\title{
Manipulation and Characterization of Molecular Scale Components
}

\author{
Islamshah Amlani \\ Physical Sciences Research \\ Laboratory, Motorola Labs \\ 7700 S. River Parkway \\ Tempe, AZ 85224 \\ (480) 755-5233 \\ Islamshah.Amlani@motorola.com
}

\author{
Ruth Zhang \\ Physical Sciences Research \\ Laboratory, Motorola Labs \\ 7700 S. River Parkway \\ Tempe, AZ 85224 \\ (480) 755-5233 \\ A783aa@email.mot.com
}

\author{
John Tresek \\ Physical Sciences Research \\ Laboratory, Motorola Labs \\ 7700 S. River Parkway \\ Tempe, AZ 85224 \\ (480) 755-5233 \\ A766aa@email.mot.com
}

\author{
Larry Nagahara \\ Physical Sciences Research Laboratory, Motorola Labs \\ 7700 S. River Parkway \\ Tempe, AZ 85224 \\ (480) 755-5233 \\ A0873aa@email.mot.com
}

\author{
Raymond K. Tsui \\ Physical Sciences Research Laboratory, Motorola Labs \\ 7700 S. River Parkway \\ Tempe, AZ 85224 \\ (480) 755-5233 \\ Ambk40@email.mot.com
}

\section{Categories and Subject Descriptors}

A.m [MISCELLANEOUS]

\section{General Terms}

Measurement, Experimentation.

\section{Keywords}

Molecular electronics, quantum devices, carbon nanotubes.

The last decade has witnessed several remarkable advances in the field of molecular electronics. Custom synthesized molecules have been shown to exhibit useful electronic functions such as switching and memory. Carbon nanotubes (CNTs), with a diameter as small as $1 \mathrm{~nm}$, have shown transistor-like properties. Meanwhile, the molecular recognition properties of DNA have been exploited to facilitate the self-assembly of nanoscale structures. Not surprisingly, molecular electronics was named "the breakthrough of the year" by Science Magazine in 2001. The unique potential of molecular electronics is the "bottom-up" self-assembly of inherently small objects such as molecules into devices and circuits. This represents a paradigm shift compared to the semiconductor "top-down" approach. Furthermore, the simplicity of this approach also offers significant economic edge over the conventional approach in which the escalating costs of wafer processing facilities are approaching tens of billions of dollars.

Permission to make digital or hard copies of all or part of this work for personal or classroom use is granted without fee provided that copies are not made or distributed for profit or commercial advantage and that copies bear this notice and the full citation on the first page. To copy otherwise, or republish, to post on servers or to redistribute to lists, requires prior specific permission and/or a fee.

$D A C^{\prime} 03$, June 2-6, 2003, Anaheim, California, USA

Copyright 2003 ACM 1-58113-688-9/03/0006 ...\$5.00.
One key aspect in molecular electronics is the ability to manipulate molecular scale components (MSCs) such as organic molecules, carbon nanotubes, nanorods, DNA molecules and nanoparticles for electrical characterization. These MSCs form the building blocks for a very advanced and highly scaled information processing system. Measurements of these MSCs and understanding their properties is crucial and will form the basis from which more complex circuits and devices may be built. Here, we will present some of the techniques that we have developed to manipulate and characterize custom synthesized electronics molecules and carbon nanotubes.

First, we will present a test platform that we have developed to facilitate the transport measurements of electronic molecules [1]. The technique consists of forming a self-assembled monolayer of the investigated molecule on pre-patterned electrodes, followed by bridging the electrodes with a nanoscale "conductive medium" such as a nanoparticle or a nanorod. Figure 1 shows the schematic and the scanning electron micrograph of the assembled structure; here, an $80 \mathrm{~nm} \mathrm{Au}$ nanoparticle is used as a conductive medium and is trapped in the gap via an AC electric field. This technique can potentially allow a quick and easy way to screen a large number of electronic molecules. We will also present preliminary transport measurements of a test molecule. 

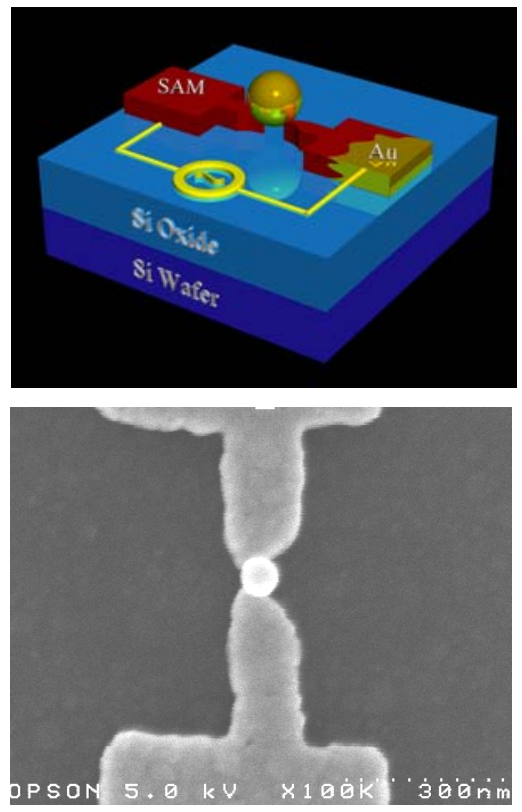

Figure 1. A Schematic and an SEM image of the test-bed for molecular electronics measurements.

Next, we will present approaches we have taken to manipulate and characterize single-walled carbon nanotubes (SWNTs) in small circuits. The first approach is similar to the one used for trapping nanoparticles in small gaps. In this technique, bulk grown SWNTs are first purified and then suspended in an appropriate solvent. SWNT assembly in between electrodes is performed by dispensing the nanotube containing solution on the electrode structure and applying an AC field (with predetermined amplitude and frequency parameters) to the electrodes [2]. Figure 2 shows an atomic force microscope image of an assembled circuit using this technique.

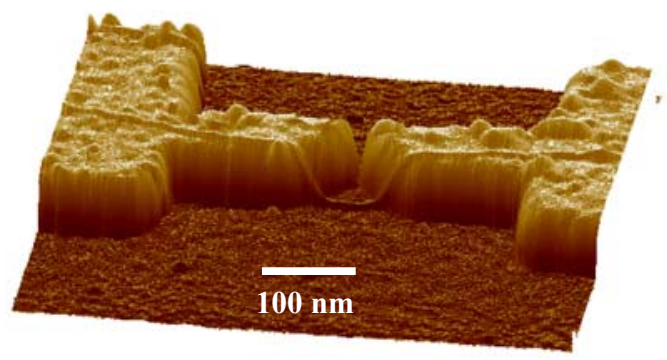

Figure 2. An AC field assembled carbon nanotube circuit.

In order to integrate SWNTs into circuits, we have also developed a single step lithographic approach to pattern catalyst islands on top of metal electrodes [3]. In this approach, a catalyst suspension is either sprayed or evaporated immediately after metal electrodes are patterned by lithography. The substrate then goes through a conventional metal lift-off process, leaving a substrate with catalyst exclusively on the desired metal pads. Chemical vapor deposition is performed on the patterned substrates to form CNTs bridging neighboring electrodes and produce a ready to test circuit. Transmission electron microscope (TEM) images demonstrate that CVD grown nanotubes are predominantly single-walled. Figure 3 shows a representative circuit and a TEM image of a SWNT. We have also developed other processes in which contact electrodes are formed post-CVD on top of SWNTs and the gap between the electrodes is tuned to as small as $100 \mathrm{~nm}$. These SWNT structures have allowed us to fabricate field-effect transistors with high gain and single-electron transistors showing Coulomb blockade at room temperature.

(a)

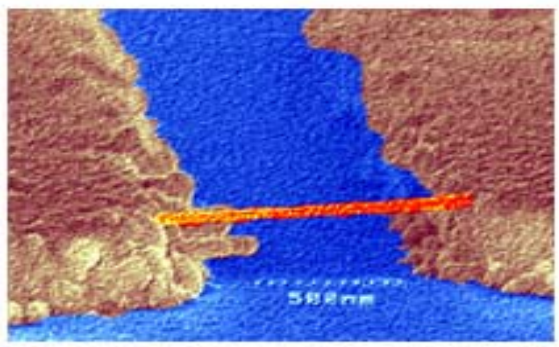

(b)

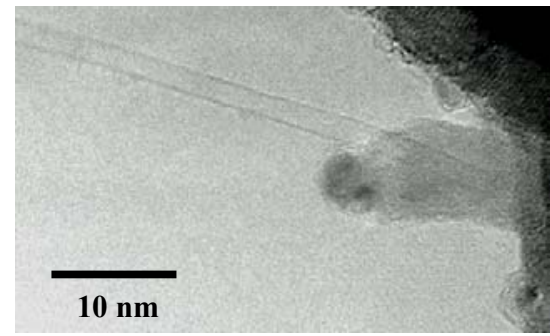

Figure 3. (a) An SEM image of a CVD grown carbon nanotube bridging a pair of electrodes. (b) TEM image of the single walled carbon nanotube.

\section{REFERENCES}

[1] Amlani, I. et al. An Approach to Transport Measurements of Electronics Molecules. Applied Physics Letters, 80 (2002), 2761-2764

[2] Nagahara, L. et al. Directed Placement of Suspended Carbon Nanotubes for Nanometer-scale Assembly. Applied Physics Letters, 80 (2002), 3826-3829.

[3] Zhang, R. et al. Formation of Carbon Nanotubes Via Reduced Pressure Chemical Vapor Deposition, Journal of Physical Chemistry (2003 ASAP Article 10.1021/jp026813m). 